01,05

\title{
Моделирование вынужденных колебаний намагниченности в системе трех ферромагнитных нанодисков
}

\author{
(C) Р.В. Горев, Е.В. Скороходов, В.Л. Миронов
}

Институт фоизики микроструктур РАН,

Нижний Новгород, Россия

E-mail: gorevrv@ipmras.ru

Поступила в Редакцию 26 марта 2020 г.

В окончательной редакции 26 марта 2020 г.

Принята к публикации 2 апреля 2020 г.

\begin{abstract}
Проведено микромагнитное моделирование ферромагнитного резонанса (ФМР) в системе, представляющей собой цилиндрический стек, состоящий из трех ферромагнитных нанодисков, в котором вследствие магнитостатического взаимодействия в равновесном состоянии реализуется геликоидальное распределение магнитных моментов. Исследованы особенности перестройки спектра ФМР и пространственной структуры резонансных мод колебаний такой системы во внешнем магнитном поле.
\end{abstract}

Ключевые слова: микромагнитное моделирование, ферромагнитный резонанс, субмикронные структуры, геликоидальное распределение.

DOI: 10.21883/FTT.2020.09.49752.22H

\section{1. Введение}

В последнее время проводятся интенсивные исследования особенностей динамики намагниченности ферромагнитных наноструктур, представляющих интерес с точки зрения создания новых приборов СВЧ-электроники, таких как датчики магнитного поля, устройства хранения информации [1-3]. Наряду с системами, находящимися в однородном магнитном состоянии [4-9], большое внимание привлекают магнитные системы с неоднородным равновесным распределением намагниченности, таким как вихрь, антивихрь [10-13], скирмион [15-19]. Использование многослойных паттернированных структур дает возможность создавать трехмерные системы с неколлинеарным распределением намагниченности. В частности, недавно нами была предложена и реализована система, состоящая из трех дисков, расположенных один над другим, в которой реализуется геликоидальное распределение магнитного момента [20,21]. Для исследования неоднородных состояний широко применяются методы, основанные на микрополосковых копланарных волноводах, и магнитно-резонансная силовая микроскопия $[12,22]$.

В настоящей работе методом микромагнитного моделирования изучаются особенности ферромагнитного резонанса в системе из трех дисков во внешнем магнитном поле. Проводится анализ перестройки спектра ФМР и модового состава резонансных колебаний при переходе данной системы между состояниями с ферромагнитным и антиферромагнитным упорядочением моментов соседних дисков, а также в состоянии с геликоидальным распределением магнитных моментов.

\section{2. Методика расчета}

Рассматриваемая система представляла собой стек соосных круглых ферромагнитных дисков диаметром 100 и толщиной $4 \mathrm{~nm}$, разделенных немагнитными прослойками толщиной $4 \mathrm{~nm}$. Микромагнитное моделирование магнитных состояний и вынужденных колебаний намагниченности данной системы проводилось в пакете Object Oriented MicroMagnetic Framework (OOMMF) [21]. В качестве материала дисков был выбран пермаллой $\mathrm{Ni}_{80} \mathrm{Fe}_{20}$ (намагниченность насыщения составляла $8 \cdot 10^{5} \mathrm{~A} / \mathrm{m}$, постоянная обменного взаимодействия $13 \cdot 10^{-12} \mathrm{~J} / \mathrm{m}$, параметр диссипации 0.01). В равновесном состоянии в такой системе реализуется геликоидальное распределение намагниченности, в котором углы между средними магнитными моментами соседних дисков составляют $135^{\circ}$ (рис. 1). При приложении внешнего магнитного поля $H=200 \mathrm{Oe}$ в данной системе реализуется состояние с антиферромагнитным упорядочением магнитных моментов, а при внешнем поле $H>1000$ Ое состояние с ферромагнитным упорядочением магнитных моментов [23].

На первом этапе система приводилась в состояние равновесия, после чего к ней прикладывалось переменное магнитное поле величиной $1 \mathrm{Oe}$, направленное параллельно плоскости дисков и перпендикулярно намагниченности диска II, а затем регистрировалась амплитуда установившихся колебаний. Частота возбуждающего СВЧ-поля изменялась в диапазоне от 0 до $15 \mathrm{GHz}$ с шагом $0.1 \mathrm{GHz}$. В качестве ФМР спектров рассчитывались частотные зависимости средней по системе амплитуды колебаний переменной составляющей намагниченности

$$
\mathbf{m}=\sqrt{m_{x}^{2}+m_{y}^{2}+m_{z}^{2}}
$$




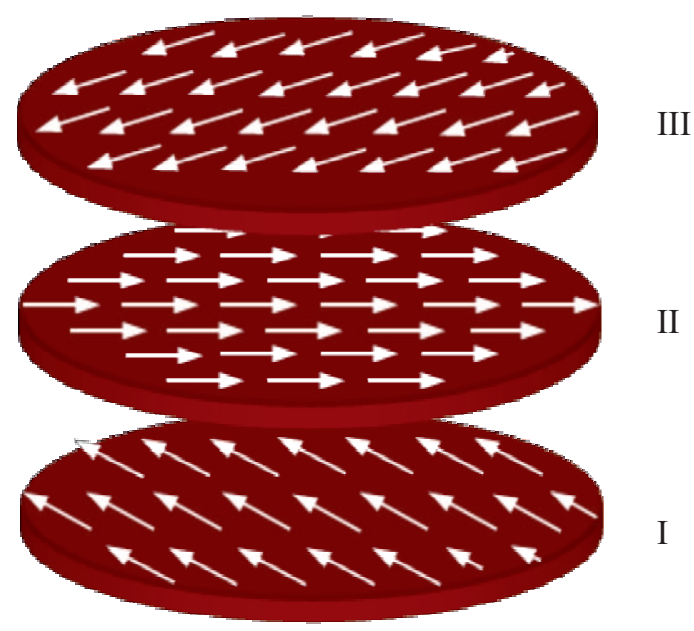

Рис. 1. Система из трех дисков с геликоидальным распределением намагниченности.

Для анализа модового состава резонансов рассчитывались пространственные распределения амплитуды колебаний всех компонент переменной составляющей намагниченности при СВЧ-накачке на резонансных часто$\operatorname{Tax}[19,20]$.

\section{3. Результаты и обсуждение}

\section{1. Состояние с ферромагнитным упорядочением}

При приложении внешнего магнитного поля $H>1000$ Ое система из трех дисков переходит в состояние с ферромагнитным упорядочением магнитных моментов дисков. Спектр колебаний намагниченности системы в этом состоянии приведен на рис. 2, $a$. Спектр содержит 2 резонансных пика. Пространственные распределения амплитуды колебаний $X$-компоненты переменной составляющей намагниченности, соответствующие данным резонансам, приведены на рис. 2, $b$. Распределения контраста в дисках соответствуют максимуму положительного отклонения $X$-компоненты намагниченности, при отрицательном отклонении контраст инвертируется.

Первому резонансу (1) соответствуют краевые моды во всех трех дисках. Максимумы амплитуды колебаний намагниченности расположены на противоположных краях каждого диска (рис. 2,b). Все колебания являются синфазными. Резонансу (2) соответствуют спин-волновые моды с тремя полуволнами. Колебания в соседних дисках являются синфазными.

\section{2. Состояние с антиферромагнитным упорядочением}

При уменьшении внешнего магнитного поля до 200 Ое система переходит в состояние с антиферромагнитным упорядочением магнитных моментов дисков.
При этом моменты дисков I и III направлены вдоль поля, диска II - против поля. Спектр колебаний для данной конфигурации системы представлен на рис. 3, a. В спектре наблюдаются четыре заметных резонансных пика (отмечены цифрами $(1)-(4))$. На рис. $3, b$ представлены пространственные распределения амплитуды резонансных колебаний $X$-компоненты переменной составляющей намагниченности.

В этом состоянии резонанс (1) соответствует квазиоднородным колебаниям в каждом из дисков. При этом колебания в разных дисках являются синфазными (различный контраст на рисунке обусловлен тем, что для дисков I и III отклонение $X$-компоненты намагниченности является отрицательным, а для диска II положительным). Резонанс (2) является суперпозицией квазиоднородной моды среднего диска и краевых мод крайних дисков. Внутри дисков I и III разность фаз между колебаниями намагниченности вблизи противоположных краев составляет 90. Разность фаз между
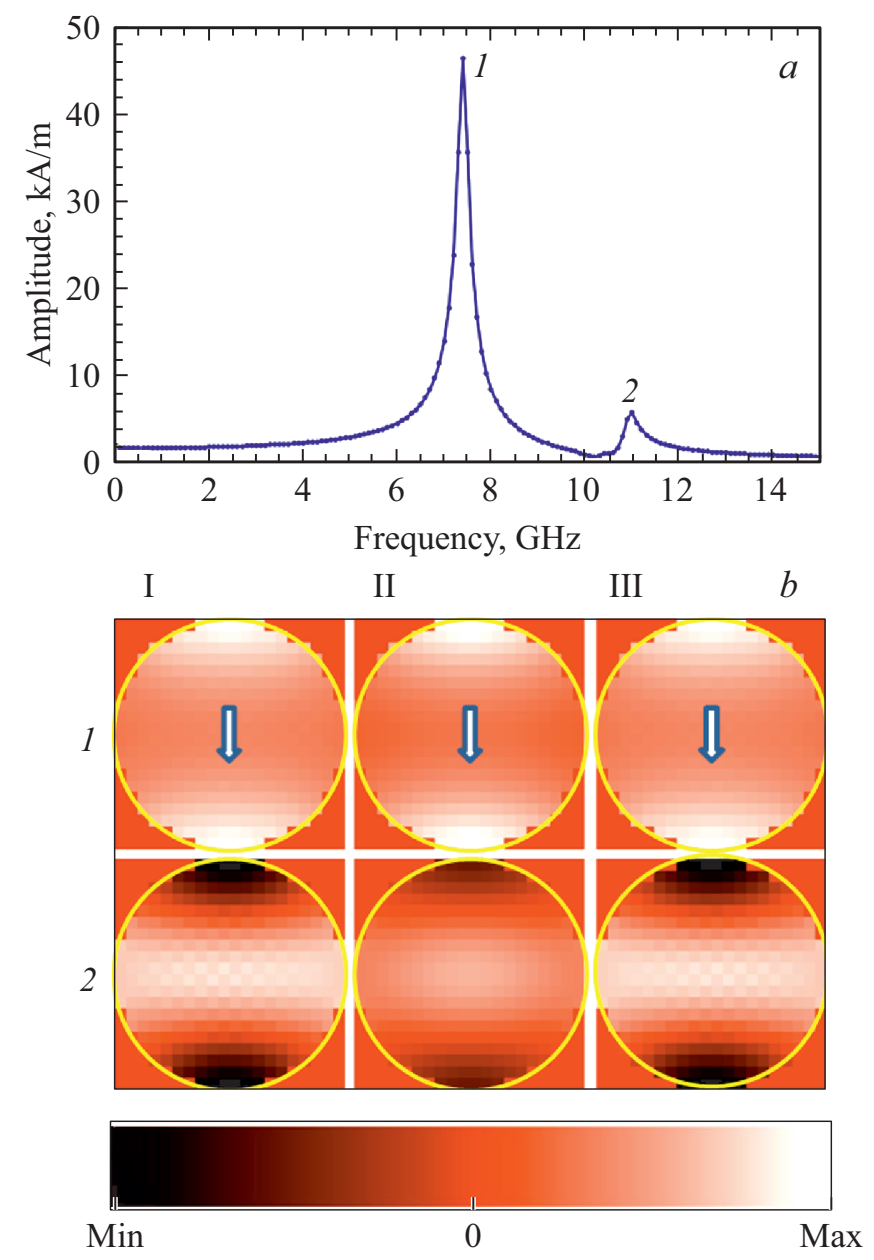

Pис. 2. $(a)-$ Спектр колебаний намагниченности системы в состоянии с ферромагнитным упорядочением магнитных моментов. $(b)$ - Нормированные пространственные распределения амплитуды колебаний $X$-компоненты переменной составляющей намагниченности, соответствующие резонансам (1) и (2). Римскими цифрами обозначены номера дисков (рис. 1). Стрелками указаны направления магнитных моментов дисков. 


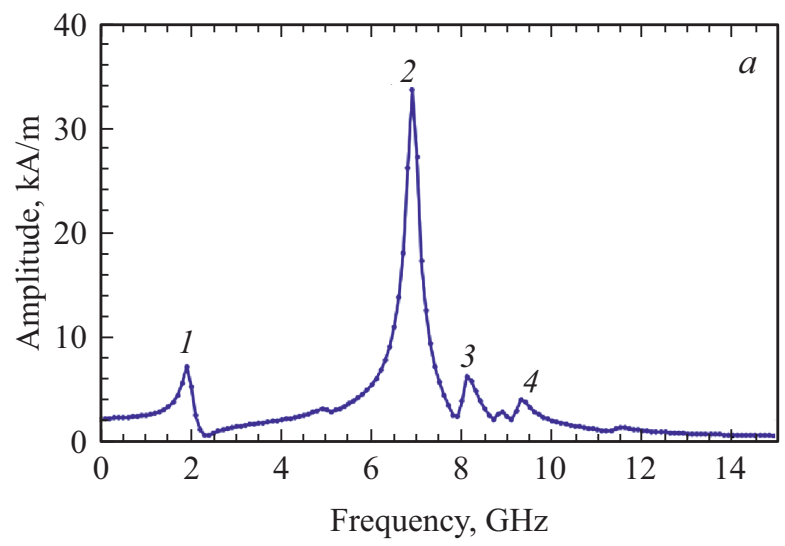

диска в области перпендикулярных диаметров. Направления, в которых происходит локализация колебаний, определяется ориентацией намагниченности в каждом из дисков. Разность фаз между колебаниями на противоположных краях диска составляет $0^{\circ}$, а между областями перпендикулярных диаметров составляет $180^{\circ}$.

\section{3. Геликоидальное состояние}

Спектр колебаний намагниченности системы в геликоидальном состоянии (внешнее поле отсутствует)

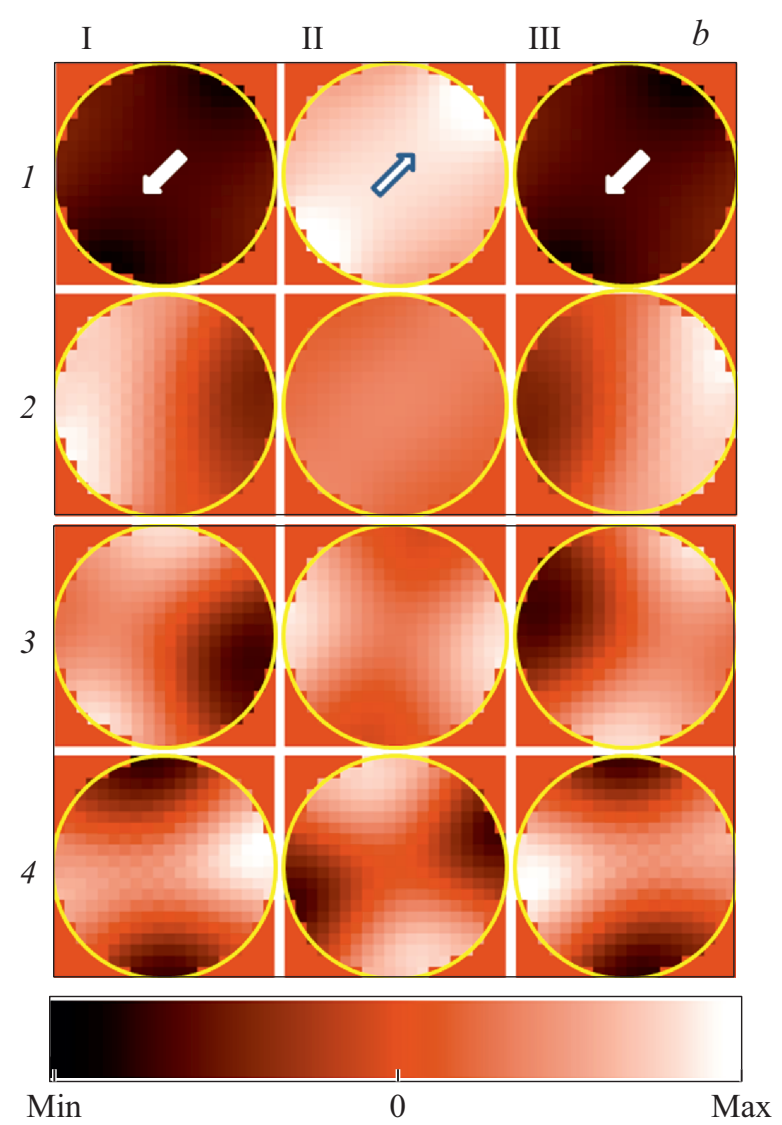

Pис. 3. (a) - Спектр колебаний намагниченности системы в состоянии с антиферромагнитным упорядочением моментов соседних дисков. $(b)$ - Пространственные распределения амплитуды резонансных колебаний $X$-компоненты переменной составляющей намагниченности. Стрелками указаны направления магнитных моментов дисков.

колебаниями в первом и третьем диске составляет $180^{\circ}$. В свою очередь, разность фаз между колебаниями в центральном и в крайних дисках составляет $90^{\circ}$.

Резонанс (3) обусловлен краевыми модами во всех дисках. В дисках I и III локализованные вблизи краев колебания находятся в противофазе, а в центральном диске (II) в фазе. Резонанс (4) соответствует неоднородными колебаниям во всех дисках, при которых максимумы и минимумы колебаний расположены вблизи края
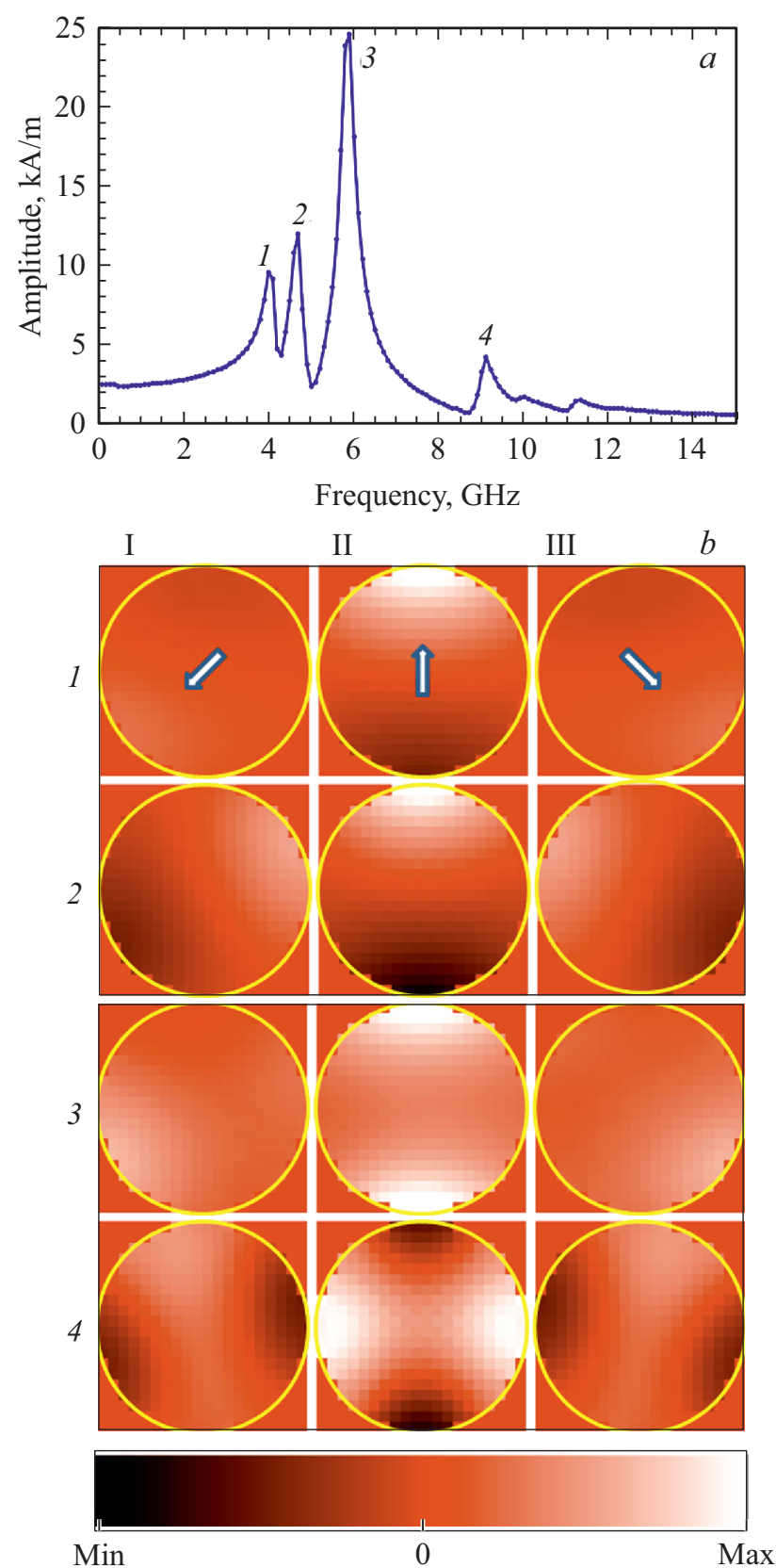

Pис. 4. (a) - Спектр колебаний намагниченности системы в геликоидальном состоянии. (b) - Пространственные распределения амплитуды колебаний $X$-компоненты переменной составляющей намагниченности. Стрелками указаны направления магнитных моментов дисков. 
приведен на рис. 4, $a$. Спектр содержит 4 интенсивных резонансных пика. Пространственные распределения амплитуды колебаний $X$-компоненты переменной составляющей намагниченности, соответствующие данным резонансам, приведены на рис. $4, b$.

Как видно из рис. $4, b$, в резонанс $(1)$ в основном дают вклад колебания намагниченности только центрального диска, которые представляют собой краевую моду. Колебания намагниченности локализованы на противоположных краях диска и имеют разность фаз $90^{\circ}$. Резонанс (2) соответствует суперпозиции краевых мод каждого диска. Разность фаз между колебаниями на противоположных краях дисков составляет также $90^{\circ}$. Резонанс (3) имеет более сложную структуру. Намагниченности I и III дисков колеблются в фазе, а колебания намагниченности диска II сдвинуты по фазе на $90^{\circ}$. Между колебаниями намагниченности у разных краев внутри I и III дисков есть разность фаз $90^{\circ}$, а у диска II разность фаз между колебаниями отсутствует. Резонанс (4) представляет собой неоднородные колебания, при которых максимумы и минимумы расположены на противоположных диаметрах. Направления, в которых происходит локализация колебаний, определяются направлением намагниченности в каждом из дисков.

Разность фаз между колебаниями намагниченности у противоположных краев диска составляет $0^{\circ}$ или $180^{\circ}$. Заметим, что амплитуда колебаний намагниченности в среднем диске (II) больше, чем в крайних дисках I и III. Это связано с тем, что во втором диске более эффективно возбуждаются магнитные колебания из-за ортогональности магнитного момента диска и магнитного СВЧ-поля накачки.

\section{4. Заключение}

Таким образом, представлены результаты численного моделирования вынужденных колебаний намагниченности в системе с сильным магнитостатическим взаимодействием, состоящей из трех ферромагнитных нанодисков, разделенных немагнитной прослойкой. Показано, что характер резонансных колебаний данной системы зависит от внешнего магнитного поля, приложенного в плоскости дисков. При намагничивании в сильном магнитном поле в системе реализуется ферромагнитное упорядочение магнитных моментов дисков и в спектре наблюдаются два резонанса, соответствующие колебаниям намагниченности, локализованным вблизи краев дисков. При уменьшении поля система переходит вначале в состояние с антиферромагнитным упорядочением магнитных моментов, а затем в состояние с геликоидальным распределением магнитных моментов, при этом происходит расщепление спектра ФМР и наблюдается формирование четырех резонансных мод коллективных колебаний намагниченности системы, что может быть изучено методами магнитно-резонансной силовой микроскопии.

\section{Финансирование работы}

Работа выполнялась при поддержке РФФИ (проект № 18-02-00247) и в рамках государственного здания (контракт № 0035-2019-0022-C-01).

\section{Конфликт интересов}

Авторы заявляют, что у них нет конфликта интересов.

\section{Список литературы}

[1] Nanomagnetism: Applications and Perspectives / Ed. C. Fermon, M. Van de Voorde. Wiley-VCH Verlag GmbH \& Co. Weinheim, Germany (2017).

[2] B. Pigeau, G. de Loubens, O. Klein, A. Riegler, F. Lochner, G. Schmidt, L.W. Molenkamp, V.S. Tiberkevich, A.N. Slavin. Appl. Phys. Lett. 96, 132506 (2010). https://doi.org/10.1063/1.3373833

[3] P.M. Braganca, B.A. Gurney, B.A. Wilson, J.A. Katine, S. Maat, J.R. Childress. Nanotechnology 21, 235202 (2010). https://doi.org/10.1088/0957-4484/21/23/235202

[4] L. Giovannini, F. Montoncello, F. Nizzoli, G. Gubbiotti, G. Carlotti, T. Okuno, T. Shinjo, M. Grimsditch. Phys. Rev. B 70, 172404 (2004). https://doi.org/10.1103/PhysRevB.70.172404

[5] G. Gubbiotti, G. Carlotti, T. Okuno, M. Grimsditch, L. Giovannini, F. Montoncello, F. Nizzoli. Phys. Rev. B 72, 184419 (2005). https://doi.org/10.1103/PhysRevB.72.184419

[6] I. Neudecker, K. Perzlmaier, F. Hoffmann, G. Woltersdorf, M. Buess, D. Weiss, C.H. Back. Phys. Rev. B 73, 134426 (2006). https://doi.org/10.1103/PhysRevB.73.134426

[7] F. Montoncello, L. Giovannini, F. Nizzoli, P. Vavassori, M. Grimsditch, T. Ono, G. Gubbiotti, S. Tacchi, G. Carlotti. Phys. Rev. B 76, 024426 (2007). https://doi.org/10.1103/PhysRevB.76.024426

[8] V.V. Naletov, G. de Loubens, G. Albuquerque, S. Borlenghi, V. Cros, G. Faini, J. Grollier, H. Hurdequint, N. Locatelli, B. Pigeau, A.N. Slavin, V.S. Tiberkevich, C. Ulysse, T. Valet, O. Klein. Phys. Rev. B 84, 224423 (2011). https://doi.org/10.1103/PhysRevB.84.224423

[9] V. Castel, J. Ben Youssef, F. Boust, R. Weil, B. Pigeau, G. de Loubens, V.V. Naletov, O. Klein, N. Vukadinovic. Phys. Rev. B 85, 184419 (2012).

[10] Р.В. Горев, В.Л. Миронов, Е.В. Скороходов. Поверхность. Рентгеновские, синхротронные и нейтронные исследования 3, 37 (2016). DOI: 10.7868/S0207352816030082

[11] Р.В. Горев, В.Л. Миронов. ФТТ 59, 11, 2154 (2017). DOI: 10.21883 FTT.2017.11.45053.10k

[12] A. Volodin, C. Van Haesendonck, E.V. Skorokhodov, R.V. Gorev, V.L. Mironov. Appl. Phys. Lett. 113, 122407 (2018). https://doi.org/10.1063/1.5040072

[13] R. Dutra, D.E. Gonzalez-Chavez, T.L. Marcondes, R.L. Sommer, S.O. Parreiras, M.D. Martins. Phys. Rev. B 99, 014413 (2019). https://doi.org/10.1103/PhysRevB.99.014413

[14] R.P. Cowburn, D.K. Koltsov, A.O. Adeyeye, M.E. Welland, D.M. Tricker. Phys. Rev. Lett. 83, 1042 (1999). https://doi.org/10.1103/PhysRevLett.83.1042 
[15] V.L. Mironov, O.L. Ermolaeva, S.A. Gusev, A.Yu. Klimov, V.V. Rogov, B.A. Gribkov, O.G. Udalov, A.A. Fraerman, R. Marsh, C. Checkley, R. Shaikhaidarov, V.T. Petrashov. Phys. Rev. B 81, 094436 (2010). https://doi.org/10.1103/PhysRevB.81.094436

[16] X.Z. Yu, Y. Onose, N. Kanazawa, J.H. Park, J.H. Han, Y. Matsui, N. Nagaosa, Y. Tokura. Nature 465, 901 (2010). DOI: 10.1038/nature09124

[17] S. Mühlbauer, B. Binz, F. Jonietz, C. Pfleiderer, A. Rosch, A. Neubauer, R. Georgii, P. Böni. Science 323, 915 (2009). DOI: $10.1126 /$ science. 1166767

[18] М.В. Сапожников, О.В. Ермолаева, Е.В. Скороходов, Н.С. Гусев, М.Н. Дроздов. Письма в ЖЭТФ 107, 378 (2018). https://doi.org/10.7868/S0370274X18060073

[19] A.A. Fraerman, B.A. Gribkov, S.A. Gusev, A.Yu. Klimov, V.L. Mironov, D.S. Nikitushkin, V.V. Rogov, S.N. Vdovichev, B. Hjorvarsson, H. Zabel. JAP 103, 073916 (2008). https://doi.org/10.1063/1.2903136

[20] M.J. Donahue, D.G. Porter. Interagency Report No. NISTIR 6376, National Institute of Standards and Technology, Gaithersburg.

[21] E.V. Skorohodov, R.V. Gorev, R.R. Yakubov, E.S. Demidov, Yu.V. Khivintsev, Yu.A. Filimonov, V.L. Mironov. J. Magn. Magn. Mater. 424, 118 (2017).

https://doi.org/10.1016/j.jmmm.2016.10.024

[22] П.Д. Ким, В.А. Орлов, В.С. Прокопенко, С.С. Замай, В.Я. Принц, Р.Ю. Руденко, Т.В. Руденко. ФТТ 57, 29 (2015).

[23] С.Н. Вдовичев, Б.А. Грибков, А.Ю. Климов, В.Л. Миронов, И.М. Нефедов, В.В. Рогов, А.А. Фраерман, И.А. Шерешевский, С.А. Гусев. Письма в ЖЭТФ 94, 418 (2011). https://doi.org/10.1134\%2FS0021364011170164

Редактор Ю.Э. Китаев 